\title{
The upgrade of the CMS ECAL Barrel calorimeter at the HL-LHC for high-precision energy and time measurements
}

\author{
Chiara Rovelli*i \\ INFN Roma \\ E-mail: chiara.rovelliecern.ch
}

\begin{abstract}
The electromagnetic calorimeter (ECAL) of the Compact Muon Solenoid experiment is currently taking data at the Large Hadron Collider (LHC). The High Luminosity phase of the LHC will provide unprecedented instantaneous and integrated luminosity, at the price of very challenging running conditions in terms of radiation level, data rates and number of simultaneous collisions in the same bunch crossing. The upgrade of the barrel part of the CMS ECAL is discussed in this paper. The requirements of the upgraded detector are reviewed and a presentation of test beam results is given.
\end{abstract}

The 39th International Conference on High Energy Physics (ICHEP2018)

4-11 July, 2018

Seoul, Korea

*Speaker.

$\dagger$ on behalf of the CMS Collaboration 


\section{Introduction}

The Compact Muon Solenoid (CMS) experiment is currently taking data at the CERN Large Hadron Collider (LHC). The CMS electromagnetic calorimeter (ECAL) [1] plays a crucial role in photon and electron reconstruction and selection, and contributes to the measurement of jets and missing transverse energy. The ECAL was designed to receive an integrated luminosity of about $500 \mathrm{fb}^{-1}$, with a peak instantaneous luminosity of $10^{34} \mathrm{~cm}^{-2} \mathrm{~s}^{-1}$. In its high luminosity phase (HL-LHC), the LHC is expected to deliver up to $4500 \mathrm{fb}^{-1}$, with a peak instantaneous luminosity of $7.5 \times 10^{34} \mathrm{~cm}^{-2} \mathrm{~s}^{-1}$ [2]. The price will be a very challenging environment, characterized by high radiation levels and high pile-up (PU) from multiple interactions per bunch crossing (up to 200). In order to maintain its excellent performance the ECAL barrel will be upgraded for the HL-LHC [3], as discussed in this paper. The ECAL endcaps will be completely replaced, because of the significant expected loss in performance due to radiation damage [4].

\section{The CMS ECAL}

ECAL is a compact homogeneous calorimeter made of $75848 \mathrm{PbW0}_{4}$ scintillating crystals. The crystals are distributed in a barrel (EB), covering the pseudorapidity region $|\eta|<1.48$, and two endcaps (EE), extending the coverage up to $|\eta|=3$. Silicon avalanche photodiodes (APDs) and vacuum phototriodes (VPTs) are used as photodetectors in the EB and EE, respectively. The signals from the photodetectors are pre-amplified and shaped by the very front-end (VFE) electronics, then digitized by a 12-bit analog-to-digital converter (ADC) running at $40 \mathrm{MHz}$ and providing a discrete set of amplitude measurements. They are stored in a front-end (FE) buffer until a Level-1 (L1) trigger is received, then transmitted to the off-detector electronics.

\section{The ECAL Barrel Upgrade}

The primary motivation for the EB upgrade [3] is the requirement in terms of latency and bandwidth of the new CMS trigger system, which will be upgraded to match the increased event rate expected at the HL-LHC. The L1 trigger latency will increase from the current $4 \mu$ s to a maximum of $12.5 \mu \mathrm{s}$, and the rate from $100 \mathrm{kHz}$ to about $750 \mathrm{kHz}$. These requirements impose the replacement of both the EB FE and off-detector electronics. The VFE cards will be also replaced to provide a better noise filtering, made necessary by the larger noise in the APDs due to the increased dark current and by the increased PU. Crystals, photodetectors, motherboards, and the mechanical structure of the ECAL barrel will stay unchanged.

\subsection{Crystals and APDs}

Several studies have been performed to evaluate the impact of the radiation expected in the HLLHC on photodetectors and crystals, and on the energy resolution of the detector. For the crystals, the main issue is the unrecoverable loss of light transmission due to the hadron irradiation [5]. Test beam and Geant 4 simulation studies have proved that the increase in the constant term of the energy resolution will be small in the barrel. Therefore the EB crystals will continue performing well and do not need to be replaced. 
For the APDs, the main concern is the increase of the dark current with integrated luminosity. The dark current is expected to reach about $100 \mu A$ at $|\eta|=0$ and about $200 \mu A$ at $|\eta|=1.45$ after an integrated luminosity of $3000 \mathrm{fb}^{-1}$, with an increase of the electronic noise by a factor 10 . To mitigate this effect, shown in Fig. 1 on the left, the EB will be operated at a temperature of $9^{\circ} \mathrm{C}$, compared to the current temperature of $18^{\circ} \mathrm{C}$. Reducing the $\mathrm{EB}$ operational temperature requires
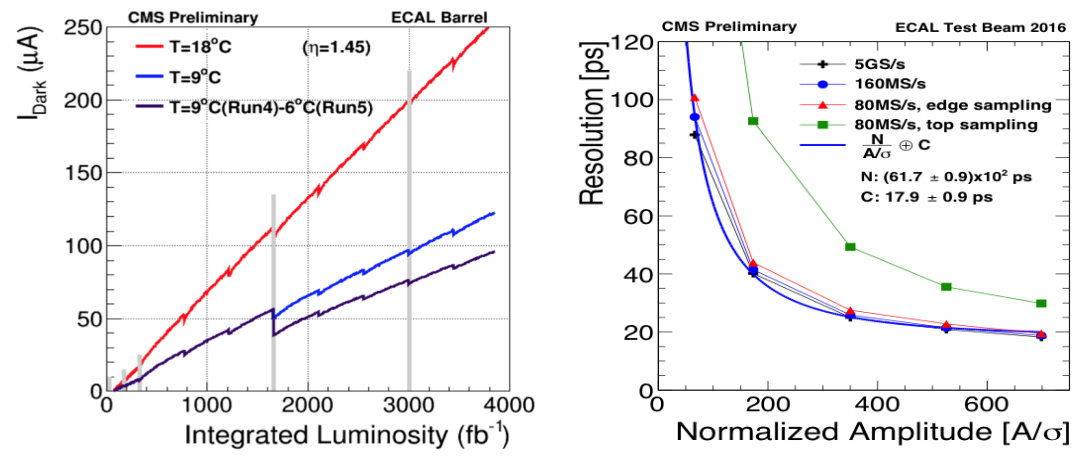

Figure 1: Left) Expected APD dark current as a function of the integrated luminosity at $|\eta|=1.45$ for operational temperatures of $18^{\circ} \mathrm{C}$ (red curve), $9^{\circ} \mathrm{C}$ (blue curve), and with a transition from $9^{\circ} \mathrm{C}$ to $6^{\circ} \mathrm{C}$ (purple curve). Right) EB timing resolution as a function of the normalized amplitude $(\mathrm{A} / \sigma)$ at different sampling frequences, obtained in an electron test beam with a TIA prototype with discrete components.

major modifications to the cooling system, which will happen during the LHC Long Shutdown 2 (2019-20).

\subsection{Electronics}

The on-detector VFE and FE cards, together with the associated low-voltage distribution system and optical links, will be fully replaced. The VFE cards will maintain a similar purpose as the current ones, with shaping time and sampling rate optimized for the HL-LHC conditions. The proposed pre-amplifier is a Trans-Impedance Amplifier (TIA), which generates a voltage image of the photocurrent and is only limited by the bandwidth of the system. Two different gains will cover the dynamic range from $50 \mathrm{MeV}$ up to $2 \mathrm{TeV}$. For the digitizer, a multi-channel ADC with 12 bit resolution and sampling rate of $160 \mathrm{MHz}$ is foreseen. The new VFE cards will have a shorter shaping time with respect to the current ones. This will significantly reduce the effect of the increased electronic noise and PU and will improve the determination of the pulse time, with a positive impact on the PU suppression and on the vertex association to electromagnetic deposits. The reduced shaping time will also allow for a better discrimination of the direct signals in the APDs ('spikes') [6], which are much faster than normal scintillation events. The spike reduction is crucial to keep the L1 rate at a sustainable level.

The connections between the front-end and the back-end electronics will be assured by new high-speed and radiation tolerant optical links (lpGBT and Versatile links), which can sustain an order of magnitude higher data transfer rate than present solutions. This removes the need for ondetector data buffering, so the L1 pipeline will be moved off-detector to accomodate the needed latency. Also the generation of the trigger primitives will happen off-detector, providing maximum granularity and flexibility. In particular, the current ECAL L1 trigger primitives consist of the sum 
of the energies in 5x5 crystal matrices and no tracking information is used, while the upgraded system will exploit the information at the single crystal level and will match electromagnetic showers to tracks [7].

The replacement of the EB electronics will require the extraction, refurbishment, and reinstallation of the detector, which will take place during the LHC Long Shutdown 3 (LS3, 2024-26).

\section{4. $R \& D$ results}

VFE and FE prototype boards are being developed and tested with laboratory and test beam measurements. Fig. 1 on the right shows the timing resolution as a function of the normalized signal amplitude (amplitude/noise RMS) for different sampling frequencies. The results were obtained at a test beam using an electron beam at the H4 line of the CERN SPS and using crystals and APDs read out by a prototype VFE with a TIA made with discrete components. A promising time resolution of $30 \mathrm{ps}$ is achieved with a sampling frequency of $160 \mathrm{MHz}$ at a noise normalized amplitude of 240. This value corresponds to an energy deposition of $25 \mathrm{GeV}$ with $100 \mathrm{MeV}$ noise per channel (HL-LHC start) and to a $50 \mathrm{GeV}$ deposition with $200 \mathrm{MeV}$ noise (HL-LHC end). Good time resolution is important for maintaining excellent physics performance in the HL-LHC environment. One of the major effects of the increased PU is a significant reduction in vertex identification efficiency, that can be recovered with the usage of precision timing.

Further prototypes will be produced and evaluated in the next years, with the goal of having the on-detector boards ready at least one year before the start of the refurbishment in LS3.

\section{Summary}

The CMS ECAL barrel will be upgraded to maintain its current performance in the challenging conditions of the HL-LHC. The crystals and APD photodetectors will be kept, while the on-detector and off-detector electronics will be replaced to accommodate higher trigger latency and to mitigate the increase in PU, noise, and spikes. In addition, a reduction in the operating temperature is foreseen to mitigate the increase in the APD dark current. The upgrade will allow ECAL to maintain its excellent energy resolution until the end of the HL-LHC, and to have the precise time resolution which is needed for pile-up mitigation in the difficult HL-LHC environment.

\section{References}

[1] CMS Collaboration, JINST 3, S08004 (2008)

[2] G. Apollinari et al., High-Luminosity Large Hadron Collider (HL-LHC): Preliminary Design Report, CERN Yellow Reports: Monographs, CERN, Geneva, 2015.

[3] CMS Collaboration, Technical Report CERN-LHCC-2017-011, Geneva, 2017.

[4] CMS Collaboration, Technical Report CERN-LHCC-2015-010, Geneva, 2015.

[5] T. Adams et al., JINST 11, P04012 (2016).

[6] D. A. Petyt, J. Phys.: Conf. Series 404, 012043 (2012).

[7] CMS Collaboration, Technical Report CERN-LHCC-2017-009, Geneva, 2017. 